\title{
Development of an Efficient Extraction Method to Quantify Microcystin-LR from Natural Microcystis Bloom Samples from the Colombo Lake, Sri Lanka
}

\author{
Idroos S.F. ${ }^{{ }^{*}}$, Welgamage A. ${ }^{2}$, Manage P.M. ${ }^{1}$, Edwards C. $^{2}$ and Lawton L. ${ }^{2}$ \\ ${ }^{1}$ Department of Zoology, Faculty of Applied Sciences, University of Sri Jayewardenepura, \\ Nugegoda, Sri Lanka \\ ${ }^{2}$ IDEAS Research Institute, Robert Gordon University, United Kingdom \\ sumaiyaidroos@gmail.com
}

\begin{abstract}
Microcystins are a diverse group of hepatotoxic secondary metabolites produced by cyanobacteria (blue-green algae). Up to date more than 90 different Microcystins were identified. Among them, Microcystin-LR (MC-LR) is one of the most toxic and most commonly encountered Microcystin variants worldwide. Although detection and extraction methods for Microcystins have been previously reported, there are no such techniques available in Sri Lanka regarding the water safety monitoring. Therefore in this study, we aim to develop an efficient extraction and quantification method of intracellular and extracellular Microcystins from natural Microcystis bloom samples. Collection of bloom material from the Colombo Lake, Sri Lanka was carried out for a period of six months from January to June 2013. Samples were filtered through GF-C filters to retain cyanobacterial cells (intracellular Microcystins) and extracellular Microcystins to pass through. Intracellular Microcystins (GFC filters) were extracted repeatedly (x2) in $80 \%$ methanol, rotary evaporated and the residue was reconstituted in 100\% methanol. Extracellular Microcystins were extracted by C18 solidphase extraction cartridges and eluted in $80 \%$ methanol. The cool-dry method carried out was similar to the GFC-filter extraction; however, the filter was subjected to heating at $55^{\circ} \mathrm{C}$ for $15 \mathrm{~min}$ followed by cooling $(15 \mathrm{~min})$ prior to the $80 \%$ methanol extraction. During the centrifugation method, 1 litre of bloom material was centrifuged $(3,000 \mathrm{~g}, 20 \mathrm{~min})$. The pellet was extracted in $100 \%$ methanol, rotary evaporated $\left(40{ }^{\circ} \mathrm{C}\right)$ and the residue was reconstituted in $80 \%$ methanol. The supernatant was solid phase extracted using C18 cartridges and eluted in $80 \%$ methanol. Identification and determination of the intracellular and extracellular Microcystin concentration were performed by photodiode array - high performance liquid chromatography. Recovery of $17.57 \pm 0.73,15.81 \pm 0.44$ and $14.66 \pm 1.04 \mathrm{ppm}$ of total microcystin-LR (intracellular and extracellular) was recorded for the filter method. In contrast, total MC-LR extracted by the cool-dry method was $0.48 \pm 0.005,0.32 \pm 0.03$ and $0.22 \pm 0.07 \mathrm{ppm}$. However, compared to other two methods, centrifugation method achieved the highest total recovery of MC-LR $(16.86 \pm 2.11,15.71 \pm 1.73$ and $13.77 \pm 0.04 \mathrm{ppm})$. Thus, it reveals that the filter method and centrifugation method are the most efficient extraction methods for MC-LR from natural bloom samples. Further optimization of these methods would enhance the monitoring and safety measures for the drinking and recreational water reservoirs in Sri Lanka.
\end{abstract}

Keywords: Microcystin-LR, Filter method, Cool-dry method, Centrifugation method, High performance liquid chromatography 